Nowak, K. (2017). Low cost retirement solutions based on robo-advisors and Exchange Traded Funds. Copernican Journal of Finance \& Accounting, 6(3), 75-94. http://dx.doi.org/10.12775/ CJFA.2017.018

\author{
KamIL NowaK ${ }^{*}$ \\ Nicolaus Copernicus University in Torun
}

\title{
LOW COST RETIREMENT SOLUTIONS BASED ON ROBO-ADVISORS AND EXCHANGE TRADED FUNDS
}

Keywords: robo advisors, ETF, retirement.

J E L Classification: G11.

Abstract: This paper briefly analyzes American retirement system and bares its shortcomings. The post crisis economic reality, low savings rates and misguided policy changes, requires searches for new retirement solutions. Especially it's worth to have a second look at costs of traditional retirement products. Analysis of mutual funds expense ratios and financial advisors fees proofs their significant impact on future retirement. New products based on ETFs and managed by robo advisors are the low cost alternative. The substance of this paper is cost comparison of traditional retirement products with innovative FinTech solutions. In a result of this analysis robo advisors and ETFs turned out to be definitely more cost effective, what makes investing more accessible and substantially increases future retirement.

\section{IIIINTRODUCTION}

It would seem that nowadays we have a knowledge and technology to provide stable and long retirement income. Utilizing years of researches combined with innovative financial instruments it should be simple to design effective retire-

Date of submission: June 4, 2017; date of acceptance: September 10, 2017.

* Contact information: k.nowak@doktorant.umk.pl, Nicolaus Copernicus University in Torun, Gagarina 13a, 87-100 Torun, Poland, phone: +48 607552 226; ORCID ID: https://orcid.org/0000-0002-8567-122X. 
ment system. Despite those assumptions looking at many countries retirement systems, it's still challenging. Analyzing American retirement system is not hard to come to a conclusion, that crisis is the best word describing it. Of course, most of the root causes of the problem lies in human nature. Low saving rates makes people more tend to spend their earnings now than save for the future. Average employees lack in financial knowledge, so shift from employer driven plans to employee's responsibility might be confusing. They don't know how much to save and how to invest. What is more, progress of medicine allows people to live longer. Longevity of life not connected with longer working period contributes to crisis as well. On the other hand, majority of crisis causes lie in macroeconomic circumstances and policy errors. Poor market returns in conjunction with high management fees obviously don't encourage to investing. What is more, US tax regulations favors Direct Benefit plans which allows large percentage of income to be deferred. When Direct Contribution plans has tax deferral limits sets so low, that retiring on tax deferred balances is not an option (Brinson \& Siegel, 2015, p. 6). Shift from DB to DC plans besides transferring investment risk from employer to employee, brought higher fees as well, because DC plans, unlike DB, invest in mutual funds, where fees are higher. Which type of plan is the most common nowadays? Figure 1 shows that it will be IRAs, despite of the fact that those plans are not sponsored by employer. Most of the IRAs assets have been rolled out from employer sponsored plans. In 2016 IRAs contribution hit 31\% of all the retirement assets. Based on that fact it can be said, that the return from IRAs have a substantial impact on their holders retirement.

Figure 1. US retirement assets 2Q 2016

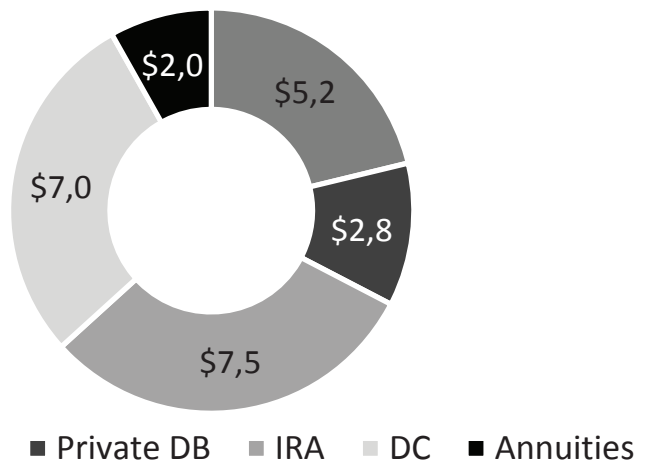

S o u r c e : author's own elaboration based on: http://us.pensiontracker.org/index.php. 
Considering that IRAs holds a bulk of Americans retirement savings it is worth to compare return rates between types of plans. Figure 2 shows geometric return rates from DB, DC and IRA plans calculated over 12 years (2000-2012). During that time so popular IRAs produced substantially lower returns that DB or DC. There are two reasons of lower returns: higher fees and assets allocation. Comparing to $4 \%$ of the assets invested in money funds by DC average IRAs invest up to $11 \%$ in those in fact safe but low return funds (Munnell et al., 2015, p. 6).

Figure 2. Geometric rates of return by plan type (2000-2012)

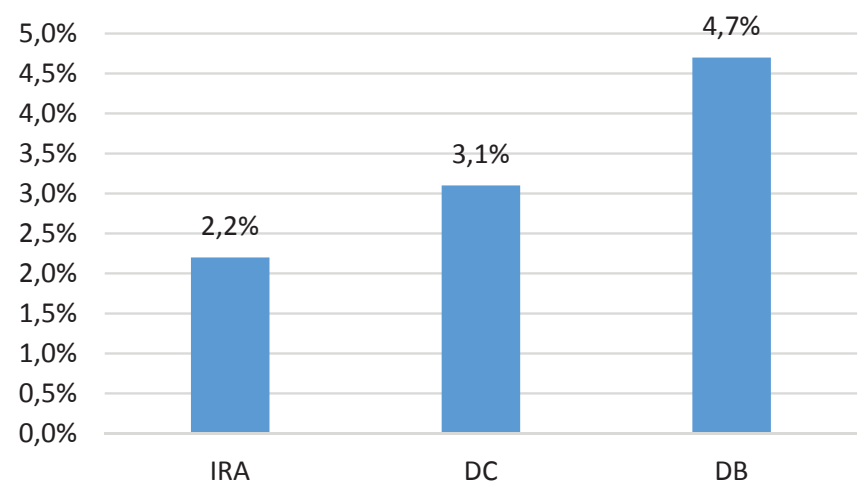

S o u r c e : Aubry, Crawford, Munnell, Investment returns: Defined Benefit vs. Defined Contribution Plans, Center for Retirement Research Boston College, 2015, p. 6.

For many years bonds were a key components of retirement portfolios. Considering goal of bringing income that allows $4-5 \%$ annual withdrawals during retirement, the bond yields were a great and safe choice until 2000, when interest rates started falling. Today 10 years US T-bond yield doesn't exceed $2 \%$. What is more dividend yields have fallen to as low as slightly more than $2 \%$ for S\&P 500 Index. Considering above mentioned conditions, conservative retirement portfolio based on bonds and dividend stocks is a good and safe solution to preserve the value of capital, but at once is very risky in terms of income point of view. If the goal of $4-5 \%$ annual withdrawals of income during retirement has to be reached, we should look for other solutions than bond and dividend stocks. Except allocation of assets, fees are other important factor that contributes to future retirement income. To understand how significant is an impact of fees on an individual retirement it's worth to quote the example brought by Er- 
icskon and Madland (Ericson \& Madland, 2014, p. 1), which demonstrates how small difference in fees results in significant difference in future retirement. Figure 3 demonstrates how big can be a difference between funds with an expense ratio $0.25 \%, 1 \%$ and $1.3 \%$ in fees paid over a lifetime by 25 years old employee earning 30,502 USD (which is a 2014 median salary in the US for that age group) and an employee earning 75000 USD. Both individuals contribute 5\% of their salary which is matched by an equal employer contribution, which results in $10 \%$. As it can be seen from the figure the difference can be as high as 124000 USD for an employee with a median salary and as high as 300000 USD for the employee earning 75000 USD. If impact of the fees on future retirement is so substantial, it's definitely worth to look for low cost retirement solutions. The main goal of this paper is to look for new low cost, performing well in a long run funds, that can be used as a core of retirement portfolios.

Figure 3. Total fees paid over lifetime by average employees

\begin{tabular}{|c|c|c|c|c|c|c|c|}
\hline \multicolumn{4}{|c|}{$\begin{array}{l}\text { Salary when worker starts saving } \\
\text { at age } 25: \$ 30,502\end{array}$} & \multicolumn{4}{|c|}{$\begin{array}{c}\text { Salary when worker starts saving } \\
\text { at age } 25: \$ 75,000\end{array}$} \\
\hline$\$ 200000$ & \multirow{2}{*}{\multicolumn{3}{|c|}{$\$ 138336$}} & \multirow{3}{*}{$\begin{array}{l}\$ 500000 \\
\$ 400000 \\
\$ 300000\end{array}$} & \\
\hline$\$ 150000$ & & & & & \multicolumn{3}{|c|}{$\$ 343147$} \\
\hline$\$ 100000$ & & & & & & & \\
\hline$\$ 50000$ & $\$ 42309$ & & & $\$ 200000$ & $\$ 10403$ & & \\
\hline ט\$ & $0,25 \%$ & $1,00 \%$ & $1,30 \%$ & \$0 & $0,25 \%$ & $1,00 \%$ & $1,30 \%$ \\
\hline
\end{tabular}

S o u r c e : author's own elaboration based on: Erickson, Madland, Fixing the drain on retirement savings, Center of American Progress, 2014, p. 3.

\section{LITERATURE REVIEW}

Exchange Traded Funds have rather a brief history. First significant studies can be dated on first decade of $21^{\text {st }}$ century. ETF's definition, description and advantages were demonstrated by Bernstein (Bernstein \& Phyllis, 2002, p. 39) and Gastineau (Gastineau, 2001), who described their main types and investigated how the operate. One of the first comparisons of ETFs and traditional mutual funds was provided by Dellva (Dellva, 2001). He presented cost benefits of ETFs. Tax advantages were studied by Poterba nad Shoven (Poterba \& Shoven, 
2002), who compared pre and after tax returns of popular index funds. Kostovetsky broadly compared ETFs and mutual funds by confronting their returns, transaction costs and expenses and tax efficiency (Kostovetsky, 2003). Since literature offers wide range of positions touching ETF topic, selection of digital advisory studies is rather poor. Robo-advisory is a recent phenomenon, that is why not many researches can be found. Fein provided an analysis of robo-advisors fiduciary standars. Advantages of digital advisory were discussed by Dapp (Dapp, 2016). Impact of recent FinTech innovations on asset management and banking business was examined by Sironi (Sironi, 2016). Digital advisory business overview can be found in numerous reports prepared by major consulting companies, like Infosys, which predicts $120 \%$ annual asset growth, Accenture sees a pressure put on traditional advisors to lower their fees a result of robo-advisory growing popularity. While Finextra states that impact of robos on present wealth advisory will be disruptive, BlackRock recognizes a gap in existing financial regulations that needs to be filled to cover digital advisory. In view of novelty of this phenomenon, there is still a space for comprehensive studies about robo-advisory.

\section{THE RESEARCH METHODOLOGY AND THE COURSE OF THE RESEARCH PROCESS}

The paper contains empirical analysis and descriptive research studies carried out in several steps. First step looks into American retirement system and identifies its shortcomings. Than taking a closer look at traditional products, their cost's impact on future retirement is measured. Thereafter advantages of ETFs and robo advisors in terms of retirement are presented. Finally, paper compares mutual funds' and ETFs return rates and expense ratios. Morningstar database has been used to find funds annual return rates and expense ratios. 10 years period has been analyzed. To determine mutual funds' ETF alternatives author used ETFdb.com Mutual Fund to ETF Converter Tool (www.etfdb. com), which utilizes mutual funds benchmarks to find the most corresponding ETFs linked to the same benchmark. The study ends with conclusions achieved by literature review and summary of provided analysis. 


\section{ETFS AS LOW COST RETIREMENT SOLUTIONS}

Since first ETFs were introduced in early nineties, their popularity grew rapidly. Nowadays ETFs became major part of asset management business and definitely the fastest growing segment of that business. They dominated indexbased investing. Looking at the massive, over $2000 \%$ growth during the last 10 years, this is just a matter of time until ETFs will overtake mutual fund industry. When it seems like exchange traded funds business is thriving, there is still a segment of the market which is still out of the reach of ETFs - retirement fund business. According to ICI investors holds only $0.02 \%$ of their $401 \mathrm{~K}$ in exchange traded funds. Accurate data for IRA is not available, but according to ICI $48 \%$ of assets is held in mutual funds, the rest in other investment vehicles including ETFs. Considering further research in this paper proving many ETFs advantages over traditional investment funds, the question arises: what are the reasons of mutual funds dominancy in retirement segment of the market? The answer is very interesting and lies in one of ETFs core advantages - intra daily trading. Most defined contribution fund provider's infrastructure isn't ready for intraday trading. The update is of course possible, but requires substantial expenses, so for the most of the industry holding status quo isn't only convenient but very profitable as well. Market examples proves that adopting ETFs to defined contribution fund business doesn't have to be expensive. Vanguard solved the problem of intraday trading by applying contributions into money market fund first and then allowing participants to convert the assets to chosen ETF when feasible. That is an example of frugal solution, but the most desired one is to develop new platform supporting intra daily trading, e.g. Charles Schwab, to allow investors fully benefit from one of many ETFs advantages. What are the other advantages? and Where does that growing popularity come from? The answer is very simple and 'exchange traded' is the key reason to unique features that differs ETFs from other investment vehicles:

- Accessibility - Markets and asset classes reserved only for large institutional investors became easily accessible for individual investors.

- Transparency -ETFs are obliged to display their portfolios on a daily basis. ETFs investors unlike mutual funds clients do not have to wonder where their funds have been invested between reporting periods or if a portfolio manager has taken unnecessary risk. Knowing the underlying assets investors may avoid doubling the exposures by holding those assets elsewhere. 
- Liquidity - 'Exchange traded' is the key to ETFs extraordinary liquidity. They can be traded multiple times, daily exactly as stocks with transparency and regulatory protection. Also like stocks ETFs can be held with a margin, shorted or optioned. The creation/redemption process allows investors to arbitrage between fund and its underlying securities.

- Tax efficiency - Unlike mutual funds ETFs do not expose their shareholders to capital gains distributions. The redemption in kind ability practically eliminates the need of capital gains distributions.

- Costs - Last but not least, probably the biggest, advantage of ETFs is their expense ratio. Average mutual fund expense ratio is almost $150 \%$ higher than average ETF's expense ratio. The savings can be achieved because ETFs are traded on the stock exchange and most of the costs like recordkeeping or sending the prospectus are borne by brokers.

In summary ETFs set up new investing standards (Nowak, 2016, p. 166). With lower costs, liquidity, accessibility and tax efficiency this isn't a surprise, that they are attracting investors money definitely faster than regular mutual funds (Hill et al., 2015, p. 9). All ETF's unique features contributed to their growing popularity, but from the retirement point of view the most important is cost effectivity. According to Morningstar, the average expense ratio for an index U.S. ETF is $0.35 \%$ and $0.87 \%$ for actively managed ETF, although many of the most popular index tracking ETFs offer ratios below $0.1 \%$. Comparing to mutual funds, which average fee is $0.65 \%$ for a passive fund and $1.21 \%$ for an active fund. Considering how even small differences in fees impact future retirement (see figure 3), that is definitely worth to take a closer look on fees and performance of ETFs and traditional mutual funds. Table 1 shows expense ratio and performance of 11 biggest mutual funds tracking S\&P 500 Index. While Table 2. Presents the same data for 3 major ETFs, tracking S\&P 500 as well. Looking at AUM, it's obvious that traditional mutual funds still dominate the market. Average index tracking mutual fund manages 27.08 billion dollars of assets. While average AUM for ETFs is not even 60 million. Of course Exchange Traded Funds are still growing segment of assets management business. Where does this fast growth come from? 
Table 1. Mutual funds tracking S\&P 500 expense ratio and performance

\begin{tabular}{|c|c|c|c|c|c|c|c|c|c|c|c|}
\hline \multirow{2}{*}{ Fund } & \multirow{2}{*}{ Ticker } & \multirow{2}{*}{$\begin{array}{c}\text { AUM } \\
\text { (millions } \\
\text { USD) }\end{array}$} & \multirow{2}{*}{$\begin{array}{c}\text { Expense } \\
\text { ratio } \\
(\%)\end{array}$} & \multicolumn{4}{|c|}{ Performance (\%) } & \multicolumn{4}{|c|}{ +/- S\&P 500 (\%) } \\
\hline & & & & $1 \mathrm{Yr}$ & $3 \mathrm{Yr}$ & $5 \mathrm{Yr}$ & $10 \mathrm{Yr}$ & $1 \mathrm{Yr}$ & $3 \mathrm{Yr}$ & $5 \mathrm{Yr}$ & $10 \mathrm{Yr}$ \\
\hline $\begin{array}{l}\text { Vanguard } 500 \\
\text { Index Investor }\end{array}$ & VFINX & 306400 & 0.14 & 21.29 & 10.14 & 13.77 & 7.53 & -0.69 & -0.12 & -0.23 & -0.12 \\
\hline $\begin{array}{l}\text { Fidelity } 500 \\
\text { Index Fund } \\
\text { Investor Class }\end{array}$ & FUSEX & 116600 & 0.10 & 21.34 & 10.21 & 13.84 & 7.58 & -0.64 & -0.06 & -0.16 & -0.07 \\
\hline $\begin{array}{l}\text { Schwab S\&P } \\
500 \text { Index }\end{array}$ & SWPPX & 25800 & 0.09 & 21.34 & 10.18 & 13.83 & 7.61 & -0.65 & -0.09 & -0.17 & -0.04 \\
\hline $\begin{array}{l}\text { BlackRock S\&P } \\
500 \text { Index Fund } \\
\text { Investor A }\end{array}$ & WFSPX & 10100 & 0.36 & 21.02 & 9.90 & 13.50 & 7.27 & -0.08 & -0.42 & -0.40 & -0.38 \\
\hline $\begin{array}{l}\text { USAA S\&P } \\
500 \text { Index } \\
\text { Fund Member } \\
\text { Shares }\end{array}$ & USSPX & 6200 & 0.25 & 21.14 & 10.01 & 13.64 & 7.40 & -0.84 & -0.25 & -0.36 & -0.25 \\
\hline $\begin{array}{l}\text { Principal } \\
\text { LargeCap S\&P } \\
500 \text { Index Fund } \\
\text { Class A }\end{array}$ & PLSAX & 5300 & 0.48 & 21.33 & 9.72 & 13.37 & 7.00 & -0.58 & -0.53 & -0.61 & -0.64 \\
\hline $\begin{array}{l}\text { TIAA-CREF } \\
\text { S\&P } 500 \text { Index } \\
\text { Retire }\end{array}$ & TRSPX & 4100 & 0.30 & 21.06 & 9.95 & 13.58 & 7.33 & -0.93 & -0.31 & -0.42 & -0.32 \\
\hline $\begin{array}{l}\text { MM S\&P 500 } \\
\text { Index Fund } \\
\text { Class A }\end{array}$ & MMFFX & 3800 & 0.72 & 20.56 & 9.51 & 13.15 & 6.93 & -0.89 & -0.79 & -0.79 & -0.72 \\
\hline $\begin{array}{l}\text { Nationwide } \\
\text { S\&P } 500 \text { Index } \\
\text { Fund Class A }\end{array}$ & GRMAX & 2600 & 0.60 & 20.71 & 9.66 & 13.28 & 7.05 & -0.74 & -0.63 & -0.66 & -0.60 \\
\hline $\begin{array}{l}\text { State Farm S\&P } \\
500 \text { Index Fund } \\
\text { Class A }\end{array}$ & SNPAX & 1400 & 0.68 & 20.62 & 9.51 & 13.10 & 6.87 & -0.83 & -0.79 & -0.84 & -0.78 \\
\hline $\begin{array}{l}\text { MainStay S\&P } \\
500 \text { Index Fund } \\
\text { Class A }\end{array}$ & MSXAX & 1300 & 0.60 & 20.75 & 9.66 & 13.27 & 7.06 & -0.70 & -0.63 & -0.67 & -0.59 \\
\hline $\begin{array}{l}\text { Invesco S\&P } \\
500 \text { Index Fund } \\
\text { Class A }\end{array}$ & SPIAX & 1000 & 0.59 & 20.75 & 9.65 & 13.29 & 7.12 & -0.70 & -0.64 & -0.65 & -0.53 \\
\hline $\begin{array}{l}\text { Deutsche S\&P } \\
500 \text { Index Fund } \\
\text { Class A }\end{array}$ & SXPAX & 940 & 0.64 & 21.23 & 9.55 & 13.25 & 6.99 & -0.77 & -0.71 & -0.74 & -0.66 \\
\hline
\end{tabular}


Table 1. Mutual funds tracking S\&P 500 expense ratio and performance

\begin{tabular}{|c|c|c|c|c|c|c|c|c|c|c|c|}
\hline \multirow{2}{*}{ Fund } & \multirow{2}{*}{ Ticker } & \multirow{2}{*}{$\begin{array}{c}\text { AUM } \\
\text { (millions } \\
\text { USD) }\end{array}$} & \multirow{2}{*}{$\begin{array}{c}\text { Expense } \\
\text { ratio } \\
(\%)\end{array}$} & \multicolumn{4}{|c|}{ Performance (\%) } & \multicolumn{4}{|c|}{ +/- S\&P 500 (\%) } \\
\hline & & & & $1 \mathrm{Yr}$ & $3 \mathrm{Yr}$ & $5 \mathrm{Yr}$ & $10 \mathrm{Yr}$ & $1 \mathrm{Yr}$ & $3 \mathrm{Yr}$ & $5 \mathrm{Yr}$ & $10 \mathrm{Yr}$ \\
\hline $\begin{array}{l}\text { State Street } \\
\text { Equity } 500 \\
\text { Index A }\end{array}$ & SSSVX & 870 & 0.47 & 20.37 & 9.58 & 13.33 & 7.25 & -1.08 & -0.71 & -0.61 & -0.41 \\
\hline $\begin{array}{l}\text { Rydex S\&P } 500 \\
\text { Fund Class A }\end{array}$ & RYSOX & 280 & 1.55 & 19.4 & 8.47 & 12.08 & 6.17 & -0.7 & -0.64 & -0.65 & -0.53 \\
\hline $\begin{array}{l}\text { QS S\&P } 500 \\
\text { Index Fund } \\
\text { Class A }\end{array}$ & SBSPX & 250 & 0.59 & 20.79 & 9.67 & 13.3 & 7.08 & -0.65 & -0.62 & -0.63 & -0.57 \\
\hline $\begin{array}{l}\text { Victory S\&P } \\
500 \text { Index Fund } \\
\text { Class A }\end{array}$ & MUXAX & 250 & 0.58 & 20.91 & 9.68 & 13.23 & 7.02 & -0.54 & -0.62 & -0.71 & -0.64 \\
\hline $\begin{array}{l}\text { PNC S\&P } 500 \\
\text { Index Fund } \\
\text { Class A }\end{array}$ & PIIAX & 160 & 0.44 & 20.81 & 9.65 & 13.32 & 7.1 & -0.63 & -0.64 & -0.62 & -0.55 \\
\hline Average & & 27075 & 0.51 & 20.86 & 9.71 & 13.34 & 7.13 & -0.70 & -0.51 & -0.55 & -0.47 \\
\hline Median & & 2000 & 0.53 & & & & & & & & \\
\hline
\end{tabular}

S o u r c e : author's own elaboration based on: Data source: Morningstar 15.03.2017.

There is as many answers as many unique features of ETFs, but one of them is expense ratio. Comparing average expense ratio of $0.51 \%$ for mutual funds and $0.06 \%$ for ETFs, the choice of conscious investor is simple. Especially considering that index tracking ETFs perform slightly better than mutual funds. In one year period the investor can earn $0.40 \%$ more investing in ETFs. Comparing performance in terms of retirement - 10 year return rate differs by $0.45 \%$. What is more, Exchange traded funds more accurately track their underlying index. During last 5 years average difference between ETFs performance and S\&P 500 is only $0.01 \%$. While mutual funds brought $0.55 \%$ less return than their underlying index. Relating those findings to retirement considerations it's hard not to come to conclusion, that index tracking ETFs are better choice than traditional mutual funds because of their cost effectiveness, performance and accuracy. 
Table 2. ETFs tracking S\&P 500 expense ratio and performance

\begin{tabular}{|c|c|c|c|c|c|c|c|c|c|c|c|}
\hline \multirow{2}{*}{ Fund } & \multirow{2}{*}{ Ticker } & \multirow{2}{*}{$\begin{array}{c}\text { AUM } \\
\text { (millions } \\
\text { USD) }\end{array}$} & \multirow{2}{*}{$\begin{array}{c}\text { Expen- } \\
\text { se ratio } \\
(\%)\end{array}$} & \multicolumn{4}{|c|}{ Performance (\%) } & \multicolumn{4}{|c|}{ +/- S\&P 500 (\%) } \\
\hline & & & & $1 \mathrm{Yr}$ & $3 \mathrm{Yr}$ & $5 \mathrm{Yr}$ & $10 \mathrm{Yr}$ & $1 \mathrm{Yr}$ & $3 \mathrm{Yr}$ & $5 \mathrm{Yr}$ & $10 \mathrm{Yr}$ \\
\hline S\&P 500 Index & & & & 21.00 & 10.28 & 13.97 & 7.66 & - & - & - & - \\
\hline $\begin{array}{l}\text { SPDR S\&P } 500 \\
\text { ETF }\end{array}$ & SPY & 210 & 0.09 & 21.35 & 10.17 & 13.84 & 7.56 & 0.35 & -0.11 & -0.13 & -0.10 \\
\hline $\begin{array}{l}\text { iShares Core } \\
\text { S\&P } 500 \text { ETF }\end{array}$ & IVV & 100 & 0.04 & 21.00 & 10.22 & 13.91 & 7.60 & 0.00 & 0.06 & 0.06 & 0.04 \\
\hline $\begin{array}{l}\text { Vanguard S\&P } \\
500 \text { ETF }\end{array}$ & VOO & 60 & 0.05 & 21.44 & 10.25 & 13.93 & - & -0.44 & 0.03 & 0.04 & - \\
\hline Average & & 123.33 & 0.06 & 21.26 & 10.21 & 13.89 & 7.58 & -0.03 & -0.01 & -0.01 & -0.03 \\
\hline Median & & 100 & 0.05 & & & & & & & & \\
\hline
\end{tabular}

S o u r c e : author's own elaboration based on: data source: Morningstar 15.03.2017.

What about other types of funds then? Tables 3, 4, 5 and 6 present most popular mutual funds and their ETF alternatives proposed by ETFdb.com Mutual Fund to ETF Converter Tool (www.etfdb.com), which utilizes mutual funds benchmarks to find the most corresponding ETFs linked to the same benchmark.

Table 3. Money market ETFs and mutual funds expense ratio and performance comparison

\begin{tabular}{|c|c|c|c|c|c|c|c|c|c|c|c|}
\hline \multirow{2}{*}{ Fund } & \multirow{2}{*}{ Ticker } & \multirow{2}{*}{$\begin{array}{c}\text { AUM } \\
\text { (millions } \\
\text { USD) }\end{array}$} & \multirow{2}{*}{$\begin{array}{c}\text { Expense } \\
\text { ratio } \\
(\%)\end{array}$} & \multicolumn{4}{|c|}{ Performance (\%) } & \multicolumn{4}{|c|}{ +/- Benchmark (\%) } \\
\hline & & & & $1 \mathrm{Yr}$ & $3 \mathrm{Yr}$ & $5 \mathrm{Yr}$ & $10 \mathrm{Yr}$ & $1 \mathrm{Yr}$ & $3 \mathrm{Yr}$ & $5 \mathrm{Yr}$ & $10 \mathrm{Yr}$ \\
\hline $\begin{array}{l}\text { Fidelity Cash } \\
\text { Reserves }\end{array}$ & FDRXX & 1360800 & 0.37 & 0.09 & 0.04 & 0.03 & 0.86 & -0.29 & -0.13 & -0.1 & 0.23 \\
\hline $\begin{array}{l}\text { JPMorgan U.S. } \\
\text { Government } \\
\text { Money Market } \\
\text { Fund }\end{array}$ & MJGXX & 149400 & 0.17 & 0.02 & 0.01 & 0.01 & 0.6 & -0.36 & -0.16 & -0.12 & -0.03 \\
\hline $\begin{array}{l}\text { GS Financial Squ- } \\
\text { are Government } \\
\text { Fund }\end{array}$ & FGTXX & 92600 & 0.18 & 0.34 & 0.14 & 0.09 & 0.72 & -0.04 & -0.03 & -0.04 & 0.09 \\
\hline $\begin{array}{l}\text { Vanguard Federal } \\
\text { Money Market } \\
\text { Fund }\end{array}$ & VMFXX & 71000 & 0.11 & 0.35 & 0.15 & 0.1 & 0.73 & -0.03 & -0.02 & -0.03 & 0.1 \\
\hline
\end{tabular}


Table 3. Money market ETFs and mutual funds expense ratio...

\begin{tabular}{|c|c|c|c|c|c|c|c|c|c|c|c|}
\hline \multirow{2}{*}{ Fund } & \multirow{2}{*}{ Ticker } & \multirow{2}{*}{$\begin{array}{l}\text { AUM } \\
\text { (millions } \\
\text { USD) }\end{array}$} & \multirow{2}{*}{$\begin{array}{c}\text { Expense } \\
\text { ratio } \\
(\%)\end{array}$} & \multicolumn{4}{|c|}{ Performance (\%) } & \multicolumn{4}{|c|}{ +/- Benchmark (\%) } \\
\hline & & & & $1 \mathrm{Yr}$ & $3 \mathrm{Yr}$ & $5 \mathrm{Yr}$ & $10 \mathrm{Yr}$ & $1 \mathrm{Yr}$ & $3 \mathrm{Yr}$ & $5 \mathrm{Yr}$ & $10 \mathrm{Yr}$ \\
\hline $\begin{array}{l}\text { Wells Fargo Go- } \\
\text { vernment Money } \\
\text { Market Fund }\end{array}$ & WFGXX & 62100 & 0.13 & 0.01 & 0.01 & 0.01 & 0.53 & -0.37 & -0.16 & -0.12 & -0.1 \\
\hline Average & & & 0.19 & 0.16 & 0.07 & 0.05 & 0.69 & -0.22 & -0.10 & -0.08 & 0.06 \\
\hline Median & & & 0.17 & & & & & & & & \\
\hline $\begin{array}{l}\text { USTREAS Treasu- } \\
\text { ry Bill Auction } \\
\text { Average } 3 \text { Month }\end{array}$ & & & & 0.38 & 0.17 & 0.13 & 0.63 & & & & \\
\hline $\begin{array}{l}\text { PIMCO Enhanced } \\
\text { Short Maturity } \\
\text { Active Exchange- } \\
\text {-Traded Fund }\end{array}$ & MINT & 6387 & 0.35 & 1.74 & 2.7 & 5.06 & - & 1.36 & 2.53 & 4.93 & - \\
\hline $\begin{array}{l}\text { iShares Short Tre- } \\
\text { asury Bond ETF }\end{array}$ & SHV & 4620 & 0.15 & 0.35 & 0.15 & 0.10 & 0.77 & -0.03 & -0.02 & -0.03 & 0.14 \\
\hline $\begin{array}{l}\text { Guggenheim } \\
\text { Enhanced Short } \\
\text { Duration ETF }\end{array}$ & GSY & 999 & 0.3 & 2.06 & 3.76 & 6.16 & & 1.68 & 3.59 & 6.03 & -0.63 \\
\hline $\begin{array}{l}\text { PowerShares } \\
\text { Treasury Collate- } \\
\text { ral Portfolio }\end{array}$ & CLTL & 370 & 0.08 & - & - & - & - & - & - & - & - \\
\hline $\begin{array}{l}\text { SPDR Barclays 1-3 } \\
\text { Month T-Bill ETF }\end{array}$ & BIL & 180 & 0.14 & 0.16 & -0.04 & -0.17 & - & -0.22 & -0.21 & -0.30 & - \\
\hline Average & & & 0.20 & 1.08 & 1.64 & 2.79 & 0.77 & 0.70 & 1.47 & 2.66 & -0.25 \\
\hline Median & & & 0.15 & & & & & & & & \\
\hline
\end{tabular}

S o u r c e : author's own elaboration based on: data source: Morningstar 15.03.2017.

As it can be seen in above table, money market funds and ETFs fees are very similar and neither of them holds the advantage. When it comes to performance it's hard to determine the winner as well. In short and medium term ETFs brings returns better than the benchmark - 3 Months T-Bills average, but in the long term mutual funds beat the benchmark and ETFs brought slightly lower return than 3 Months T-Bills. In terms of portfolio building, money market funds are rather used in short term, so considering comparable expense ratios, both mutual funds and ETFs can be utilized. 
Table 4. MSCI US Broad Market Index ETFs and mutual fund expense ratio and performance comparison

\begin{tabular}{|c|c|c|c|c|c|c|c|c|c|c|c|}
\hline \multirow{2}{*}{ Fund } & \multirow{2}{*}{ Ticker } & \multirow{2}{*}{$\begin{array}{l}\text { AUM } \\
\text { (millions } \\
\text { USD) }\end{array}$} & \multirow{2}{*}{$\begin{array}{c}\text { Expense } \\
\text { ratio } \\
(\%)\end{array}$} & \multicolumn{4}{|c|}{ Performance (\%) } & \multicolumn{4}{|c|}{ +/- Benchmark (\%) } \\
\hline & & & & $1 \mathrm{Yr}$ & $3 \mathrm{Yr}$ & $5 \mathrm{Yr}$ & $10 \mathrm{Yr}$ & $1 \mathrm{Yr}$ & $3 \mathrm{Yr}$ & $5 \mathrm{Yr}$ & $10 \mathrm{Yr}$ \\
\hline $\begin{array}{l}\text { Vanguard Total } \\
\text { Stock Mkt Idx Inv }\end{array}$ & VTSMX & 541300 & 0.15 & 22.55 & 9.44 & 13.59 & 7.74 & 4.73 & -0.07 & 0.32 & 0.21 \\
\hline $\begin{array}{l}\text { Fidelity Total } \\
\text { Market Index } \\
\text { Investor }\end{array}$ & FSTMX & 40300 & 0.1 & 17.67 & 9.32 & 13.09 & 7.38 & -0.15 & -0.19 & -0.18 & -0.15 \\
\hline $\begin{array}{l}\text { Schwab Total } \\
\text { Stock Market } \\
\text { Index }\end{array}$ & SWTSX & 5700 & 0.09 & 17.67 & 9.35 & 13.13 & 7.48 & -0.15 & -0.16 & -0.14 & -0.05 \\
\hline $\begin{array}{l}\text { BlackRock Total } \\
\text { Stock Market } \\
\text { Index Inv A }\end{array}$ & BASMX & 596 & 0.29 & 17.40 & - & - & - & -0.42 & - & - & - \\
\hline $\begin{array}{l}\text { Wilshire } 5000 \\
\text { Index Fund }\end{array}$ & WFIVX & 184 & 0.67 & 17.05 & 9.03 & 12.62 & 6.77 & -0.77 & -0.48 & -0.65 & -0.76 \\
\hline Average & & & 0.26 & 18.468 & 9.285 & 13.11 & 7.34 & 0.64 & -0.22 & -0.16 & -0.19 \\
\hline Median & & & 0.15 & & & & & & & & \\
\hline $\begin{array}{l}\text { MSCI US Broad } \\
\text { Market Index }\end{array}$ & & & & 17.82 & 9.51 & 13.27 & 7.53 & & & & \\
\hline $\begin{array}{l}\text { Vanguard Total } \\
\text { Stock Market ETF }\end{array}$ & VTI & 76020 & 0.05 & 17.68 & 9.40 & 13.20 & 7.74 & -0.14 & -0.11 & -0.07 & 0.21 \\
\hline $\begin{array}{l}\text { iShares Edge } \\
\text { MSCI Min Vol } \\
\text { USA ETF }\end{array}$ & USMV & 12330 & 0.15 & 13.66 & 12.57 & 13.73 & - & -4.16 & 3.06 & 0.46 & - \\
\hline $\begin{array}{l}\text { Schwab U.S. Bro- } \\
\text { ad Market ETF }\end{array}$ & $\mathrm{SCHB}$ & 8510 & 0.03 & 20.70 & 10.29 & 13.37 & - & 2.88 & 0.78 & 0.10 & - \\
\hline $\begin{array}{l}\text { iShares Core S\&P } \\
\text { Total U.S. Stock } \\
\text { Market ETF }\end{array}$ & ITOT & 7860 & 0.03 & 17.59 & 9.83 & 13.25 & 7.33 & -0.23 & 0.32 & -0.02 & -0.20 \\
\hline $\begin{array}{l}\text { iShares Russell } \\
3000 \text { ETF }\end{array}$ & IWV & 7410 & 0.2 & 17.55 & 9.31 & 13.09 & 7.20 & -0.27 & -0.20 & -0.18 & -0.33 \\
\hline Average & & & 0.09 & 17.44 & 10.28 & 13.33 & 7.42 & -0.38 & 0.77 & 0.06 & -0.11 \\
\hline Median & & & 0.05 & & & & & & & & \\
\hline
\end{tabular}

S o u r c e : author's own elaboration based on: data source: Morningstar 15.03.2017.

Similar conclusions can be drawn from analysis of ETFs and mutual funds, which are tracking MSCI US Broad Market Index regarding the performance trends. In a long term both stays slightly below benchmark level, but it can be 
stated that both accurately tracks MSCI US Broad Market Index. However, average expenses of ETFs are significantly lower than their corresponding mutual funds, so in terms of cost effectiveness in this category ETFs are dominating.

Table 5. Barclays Capital U.S. Aggregate Bond Index ETFs and mutual fund expense ratio and performance comparison

\begin{tabular}{|c|c|c|c|c|c|c|c|c|c|c|c|}
\hline \multirow{2}{*}{ Fund } & \multirow{2}{*}{ Ticker } & \multirow{2}{*}{$\begin{array}{l}\text { AUM } \\
\text { (millions } \\
\text { USD) }\end{array}$} & \multirow{2}{*}{$\begin{array}{c}\text { Expense } \\
\text { ratio } \\
(\%)\end{array}$} & \multicolumn{4}{|c|}{ Performance (\%) } & \multicolumn{4}{|c|}{ +/- Benchmark (\%) } \\
\hline & & & & $1 \mathrm{Yr}$ & $3 \mathrm{Yr}$ & $5 \mathrm{Yr}$ & $10 \mathrm{Yr}$ & $1 \mathrm{Yr}$ & $3 \mathrm{Yr}$ & $5 \mathrm{Yr}$ & $10 \mathrm{Yr}$ \\
\hline $\begin{array}{l}\text { Vanguard Total } \\
\text { Bond Market II }\end{array}$ & VTBIX & 120900 & 0.09 & 0.45 & 2.26 & 1.86 & - & -0.31 & -0.44 & -0.47 & - \\
\hline $\begin{array}{l}\text { American Cen- } \\
\text { tury Diversified } \\
\text { Bond Inv }\end{array}$ & ADFIX & 6500 & 0.6 & 0.88 & 2.51 & 2.2 & 4.43 & 0.12 & -0.19 & -0.13 & 0.18 \\
\hline $\begin{array}{l}\text { Loomis Sayles In- } \\
\text { vestment Grade } \\
\text { Bond A }\end{array}$ & LIGRX & 6100 & 0.85 & 5.32 & 1.6 & 3.08 & 5.52 & 4.56 & -1.1 & 0.75 & 1.27 \\
\hline $\begin{array}{l}\text { Russell Inv Stra- } \\
\text { tegic Bond A }\end{array}$ & RFDAX & 5000 & 0.96 & 1.32 & 2.55 & 2.59 & 4.25 & 0.56 & -0.15 & 0.26 & 0 \\
\hline $\begin{array}{l}\text { Wells Fargo Core } \\
\text { Bond A }\end{array}$ & MBFAX & 4900 & 0.78 & 0.73 & 2.41 & 2.37 & 4.6 & -0.03 & -0.29 & 0.04 & 0.35 \\
\hline Average & & & 0.66 & 1.74 & 2.27 & 2.42 & 4.70 & 0.98 & -0.43 & 0.09 & 0.45 \\
\hline Median & & & 0.78 & & & & & & & & \\
\hline $\begin{array}{l}\text { Barclays Capital } \\
\text { U.S. Aggregate } \\
\text { Bond Index }\end{array}$ & & & & 0.76 & 2.7 & 2.33 & 4.25 & & & & \\
\hline $\begin{array}{l}\text { iShares Core U.S. } \\
\text { Aggregate Bond } \\
\text { ETF }\end{array}$ & AGG & 42260 & 0.05 & 0.21 & 2.16 & 1.97 & 3.93 & -0.55 & -0.54 & -0.36 & -0.32 \\
\hline $\begin{array}{l}\text { Vanguard Total } \\
\text { Bond Market ETF }\end{array}$ & BND & 32200 & 0.06 & 0.58 & 2.15 & 1.99 & - & -0.18 & -0.55 & -0.34 & - \\
\hline $\begin{array}{l}\text { Schwab U.S. } \\
\text { Aggregate Bond } \\
\text { ETF }\end{array}$ & $\mathrm{SCHZ}$ & 3410 & 0.04 & 0.14 & 2.11 & 1.89 & - & -0.62 & -0.59 & -0.44 & - \\
\hline Average & & & 0.05 & 0.31 & 2.14 & 1.95 & 3.93 & -0.45 & -0.56 & -0.38 & -0.32 \\
\hline Median & & & 0.05 & & & & & & & & \\
\hline
\end{tabular}

S o u r c e : author's own elaboration based on: data source: Morningstar 15.03.2017.

Comparing funds linked to Barclays Capital U.S. Aggregate Bond Index, ETFs still have significantly lower fees. When it comes to performance and ac- 
curacy, mutual funds stay closer to the benchmark and even outperfome it in a long run.

Table 6. MSCI All Country World ex-U.S. Index ETFs and mutual fund expense ratio and performance comparison

\begin{tabular}{|c|c|c|c|c|c|c|c|c|c|c|c|}
\hline \multirow{2}{*}{ Fund } & \multirow{2}{*}{ Ticker } & \multirow{2}{*}{$\begin{array}{l}\text { AUM } \\
\text { (millions } \\
\text { USD) }\end{array}$} & \multirow{2}{*}{$\begin{array}{c}\text { Expense } \\
\text { ratio } \\
(\%)\end{array}$} & \multicolumn{4}{|c|}{ Performance (\%) } & \multicolumn{4}{|c|}{ +/- Benchmark (\%) } \\
\hline & & & & $1 \mathrm{Yr}$ & $3 \mathrm{Yr}$ & $5 \mathrm{Yr}$ & $10 \mathrm{Yr}$ & $1 \mathrm{Yr}$ & $3 \mathrm{Yr}$ & $5 \mathrm{Yr}$ & $10 \mathrm{Yr}$ \\
\hline $\begin{array}{l}\text { Vanguard Total } \\
\text { Intl Stock Index } \\
\text { Inv }\end{array}$ & VGTSX & 251600 & 0.18 & 14.72 & 2.06 & 4.73 & 1.24 & 0.26 & 0.45 & 0.38 & -0.08 \\
\hline $\begin{array}{l}\text { Harbor Interna- } \\
\text { tional Investor }\end{array}$ & HIINX & 34500 & 1.14 & 8.36 & -0.64 & 3.31 & 2.09 & -6.1 & -2.25 & -1.04 & 0.77 \\
\hline $\begin{array}{l}\text { Oakmark Interna- } \\
\text { tional Investor }\end{array}$ & OAKIX & 29500 & 1 & 22.68 & 3.07 & 8.83 & 4.55 & 8.22 & 1.46 & 4.48 & 3.23 \\
\hline $\begin{array}{l}\text { Fidelity Inter- } \\
\text { national Index } \\
\text { Investor }\end{array}$ & FSIIX & 16300 & 0.2 & 12.32 & 1.29 & 5.75 & 1.04 & -2.14 & -0.32 & 1.4 & -0.28 \\
\hline $\begin{array}{l}\text { American Funds } \\
\text { Intl Gr and Inc A }\end{array}$ & IGAAX & 11200 & 0.91 & 12.36 & -0.47 & 4.85 & - & -2.1 & -2.08 & 0.5 & - \\
\hline Average & & & 0.69 & 14.09 & 1.06 & 5.49 & 2.23 & -0.37 & -0.55 & 1.14 & 0.91 \\
\hline Median & & & 0.91 & & & & & & & & \\
\hline $\begin{array}{l}\text { MSCI All Country } \\
\text { World ex-U.S. } \\
\text { Index }\end{array}$ & & & & 14.46 & 1.61 & 4.35 & 1.32 & & & & \\
\hline $\begin{array}{l}\text { iShares MSCI } \\
\text { ACWI ex U.S. ETF }\end{array}$ & ACWX & 2070 & 0.33 & 12.27 & 1.14 & 3.59 & - & -2.19 & -0.47 & -0.76 & - \\
\hline $\begin{array}{l}\text { SPDR MSCI ACWI } \\
\text { ex-US ETF }\end{array}$ & CWI & 1210 & 0.3 & 12.77 & 1.77 & 4.07 & 1.83 & -1.69 & 0.16 & -0.28 & 0.51 \\
\hline Average & & & 0.56 & 12.52 & 1.46 & 3.83 & 1.58 & -1.94 & -0.16 & -0.52 & 0.51 \\
\hline Median & & & 0.32 & & & & & & & & \\
\hline
\end{tabular}

S o u r c e : author's own elaboration based on: data source: Morningstar 15.03.2017.

Going back to equity funds, but outside the U.S market this time, once more it can be noticed that expenses of ETFs are lower than mutual funds. Average ETFs fees are $0.13 \%$ lower than mutual funds. To sum up above analysis, US market and international equity ETFs expense ratio is usually significantly lower than their corresponding mutual funds. Bond ETFs fees are hard to beat as well. While in case of money market, both types of investment vehicles 
are competitive in terms of expense ratio. Based on figure 3, which shows how a small difference in fees can impact future retirement, choosing ETFs instead of traditional mutual funds to compose long term retirement portfolio, can bring significant savings. Going further, retirement portfolio based on ETFs will result in higher retirement than portfolio based on mutual funds.

\section{RISE OF ROBO-ADVISORS}

Technology has a huge impact on all the industries including finance. Implementation of FinTech solutions resulted in rise of robo-advisors, which is a common term for digital investment advisory. In other words, it's automated investment advisory and portfolio management service with minimal, or no human intervention. Robo-advisors provide internet services using algorithmic portfolio strategies. Based on online questionnaire filled out by an investor, the algorithm assesses customer's risk tolerance and gathers information to understand client's needs and investment preferences. Knowing user's investment goals and risk profile, assets allocation can be made and portfolio recommendation are presented. After the investment is placed, digital advisors offer portfolio management as well. Customer receives frequently updates, alerts and market research. To adjust to investment goals and client's preferences, algorithm provides periodically portfolio review and rebalancing when needed. Robo-advisory is a fast growing segment of assets management business. Regarding to statista.com (www.statista.com) business of robos will grow from 500 bn US dollars in 2016 to 2.2 trillion US dollars in 2020. Booming popularity comes from four major benefits of digital advice:

- Efficient investment management - robo advisory platforms are based on algorithms that provide automated portfolio rebalancing. No customer action is required. What is more, automation eliminates common human investment decision's biases.

- Transparency and accessibility - digital advisory platforms are transparent and user friendly. Internet platforms or even mobile applications allows to track the investments 24/7. Even not very sophisticated investors are able to analyze the return and compare it vs benchmark.

- Diversification - system captures the customers' investment objectives, time horizon and attitude to risk. Based on investors' preferences algorithm adheres to modern portfolio theory which manages risk exposure through portfolio diversification, investing in low costs ETFs. 
- Lower fees and lower minimum investment requirements than traditional asset managers attract investors, who don't qualify to be served by traditional institutions (France-Massey, 2016, p. 29).

Robos' fees are significantly lower than traditional asset managers. Typically, all the expenses are not higher than $1 \%$ of AUM, but same advisors offer fees as low as zero. How is it possible to charge customers with so low fees and still keep the business profitable? Of course thanks to technology, which not only empowers portfolio allocations and investment management, but impacts record keeping, compliance reviews and periodical rebalancing. Processes, which in case of traditional asset management company represent significant share of operation costs, can be fully automated and fewer personnel is required. Thanks to significant cost reduction digital advisors can operate at much lower costs and those savings can be passed on to the investors (Xia, 2016). Another advantage of robo advisors, which attracts customers is transparency of the fees. Investors have to pay only one annual management fee and ETF fees on the assets they hold. Table 7 compares 10 top US robo advisors. Breaking the list by assets, it is obvious that, 2 firms are dominating the market, both owned by traditional asset managers - Vanguard and Schwab. What is more, the sixth biggest firm Future Advisors belongs to BlackRock. All of the robos offers mostly ETFs of well known providers. Most of the portfolios are rebalanced automatically on contribution, withdrawal, dividends and whenever allocations drift from their target. Setting up the account is easy. All of the platforms allows to open the account online. Minimum balance required to open the account can be as low as $\$ 0$ for some advisors. What makes investing even more accessible for beginner investors. 'Fees' is of course the most interesting column. They vary from $0.1 \%$ to $0.5 \%$. It can be noticed that higher fees are usually correlated with high human interference, so savvy investors should choose rather fully automated platforms. Tax-loss harvesting may be as important as the fees, because when provided on daily basis, it can boost annual return by even $2 \%$. On the other hand, Table 8 presents average financial advisor fees. Of course they are much higher than robos'. FA's fees can decline below $1 \%$ only for larger than 1 million USD. 
Table 7. Top US Robo advisors characteristics

\begin{tabular}{|c|c|c|c|c|c|c|c|}
\hline Firm & $\begin{array}{l}\text { AUM } \\
\text { (millions } \\
\text { USD) }\end{array}$ & $\begin{array}{c}\text { Human } \\
\text { touch }\end{array}$ & Portfolio & Rebalancing & $\begin{array}{l}\text { Tax-loss } \\
\text { harvesting }\end{array}$ & Fees & $\begin{array}{l}\text { Account } \\
\text { minimum }\end{array}$ \\
\hline $\begin{array}{l}\text { Vanguard } \\
\text { Personal } \\
\text { Advisor } \\
\text { Services }\end{array}$ & 47,000 & High & $\begin{array}{l}\text { Vanguard mutual } \\
\text { funds, ETFs }\end{array}$ & Quarterly & $\begin{array}{l}\text { On case by } \\
\text { case basis }\end{array}$ & $\begin{array}{l}0,30 \% \text { for portfo- } \\
\text { lios below } \$ 5 \mathrm{M} \text {, } \\
\text { above } \$ 5 \mathrm{M} \text { fees } \\
\text { drop by tier, low- } \\
\text { est fee } 0,05 \% \text { for } \\
\text { portfolios larger } \\
\text { than } \$ 25 \mathrm{M}\end{array}$ & $\$ 50,000$ \\
\hline $\begin{array}{l}\text { Schwab } \\
\text { Intel- } \\
\text { ligent } \\
\text { Portfolios }\end{array}$ & 12,300 & Low & $\begin{array}{l}\text { Schwab and third } \\
\text { party ETFs }\end{array}$ & Automatic, daily & $\begin{array}{l}\text { For ac- } \\
\text { counts of } \\
\$ 50,000 \text { or } \\
\text { more }\end{array}$ & $\begin{array}{l}0,1 \% \text { under } \\
\$ 100 \mathrm{M} \\
\text { no charge above } \\
\$ 100 \mathrm{M}\end{array}$ & $\$ 5,000$ \\
\hline $\begin{array}{l}\text { Better- } \\
\text { ment }\end{array}$ & 6,700 & Low & $\begin{array}{l}\text { Vanguard and } \\
\text { iShares ETFs }\end{array}$ & $\begin{array}{l}\text { Automatic, } \\
\text { whenever alloca- } \\
\text { tions drift } 3 \% \\
\text { from the target }\end{array}$ & $\begin{array}{l}\text { Daily on all } \\
\text { accounts }\end{array}$ & $0,15-0,35 \% \ldots$ & $\$ 0$ \\
\hline $\begin{array}{l}\text { Wealth- } \\
\text { front }\end{array}$ & 4,348 & Low & $\begin{array}{l}\text { Vanguard, } \\
\text { Schwab, iShares } \\
\text { and SPDR ETFs }\end{array}$ & $\begin{array}{l}\text { Automatic, } \\
\text { whenever alloca- } \\
\text { tions drift from } \\
\text { the target }\end{array}$ & $\begin{array}{l}\text { Daily on all } \\
\text { accounts }\end{array}$ & $\begin{array}{l}\text { No charge for } \\
\text { first } \$ 10,000 \\
\text { Above } \$ 10,000 \\
\text { flat } 0,25 \%\end{array}$ & $\$ 500$ \\
\hline $\begin{array}{l}\text { Personal } \\
\text { Capital }\end{array}$ & 2,835 & High & $\begin{array}{l}\text { ETFs and for } \\
\text { portfolios above } \\
\$ 100,000 \text { indi- } \\
\text { vidual stocks }\end{array}$ & On all accounts & $\begin{array}{l}\text { On all ac- } \\
\text { counts }\end{array}$ & $0,49-0,89 \%$ & $\$ 25,000$ \\
\hline $\begin{array}{l}\text { Future } \\
\text { Advisor }\end{array}$ & 969 & $\begin{array}{l}\text { Me- } \\
\text { dium }\end{array}$ & $\begin{array}{l}\text { iShares, Van- } \\
\text { guard and SPDR } \\
\text { ETFs }\end{array}$ & $\begin{array}{l}\text { 4-6 times an- } \\
\text { nually }\end{array}$ & $\begin{array}{l}\text { On all ac- } \\
\text { counts }\end{array}$ & $0,50 \%$ & $\$ 10,000$ \\
\hline $\begin{array}{l}\text { Rebal- } \\
\text { ance IRA }\end{array}$ & 403 & High & $\begin{array}{l}\text { Vanguard, } \\
\text { iShares and SPDR } \\
\text { ETFs }\end{array}$ & Automatic & $\begin{array}{l}\text { On all ac- } \\
\text { counts }\end{array}$ & $0,50 \%$ & $\$ 100,000$ \\
\hline Acorns & 257 & Low & $\begin{array}{l}\text { Vanguard and } \\
\text { iShares ETFs }\end{array}$ & $\begin{array}{l}\text { Automatic, } \\
\text { whenever alloca- } \\
\text { tions drift } 5 \% \\
\text { from the target }\end{array}$ & No & $\begin{array}{l}\$ 1 \text { monthly } \\
\text { below } \$ 5,000 \\
\text { above } \$ 5,000 \text { flat } \\
0,25 \% \\
\text { no fees for col- } \\
\text { lege students }\end{array}$ & $\$ 0$ \\
\hline $\begin{array}{l}\text { Etrade } \\
\text { Adaptive } \\
\text { Portfolio }\end{array}$ & 180 & Low & $\begin{array}{l}\text { Vanguard, } \\
\text { iShares and SPDR } \\
\text { ETFs, active } \\
\text { mutual funds }\end{array}$ & Automatic, daily & No & $0,30 \%$ & $\$ 10,000$ \\
\hline SigFig & 114 & Low & $\begin{array}{l}\text { Vanguard, } \\
\text { iShares and } \\
\text { Schwab ETFs }\end{array}$ & Automatic, daily & $\begin{array}{l}\text { On all ac- } \\
\text { counts }\end{array}$ & $\begin{array}{l}\text { No charge below } \\
\$ 10,000 \\
\text { Above } \$ 10,000 \\
\text { flat } 0,25 \%\end{array}$ & $\$ 2,000$ \\
\hline
\end{tabular}

S o u r c e : author's own elaboration based on: data source: www.etf.com (accessed:15.03.2017). 
Table 8. Average Financial Advisor Fees

\begin{tabular}{|c|c|}
\hline \hline Investment Amounts & Average Advisor Fees (\%) \\
\hline \hline$\$ 50,000$ & $1.18 \%$ \\
\hline$\$ 100,000$ & $1.12 \%$ \\
\hline$\$ 150,000$ & $1.09 \%$ \\
\hline$\$ 250,000$ & $1.07 \%$ \\
\hline$\$ 500,000$ & $1.05 \%$ \\
\hline$\$ 1,000,000$ & $1.02 \%$ \\
\hline$\$ 1,500,000$ & $0.94 \%$ \\
\hline$\$ 2,000,000$ & $0.91 \%$ \\
\hline$\$ 2,500,000$ & $0.88 \%$ \\
\hline$\$ 5,000,000$ & $0.84 \%$ \\
\hline$\$ 7,500,000$ & $0.77 \%$ \\
\hline$\$ 10,000,000$ & $0.69 \%$ \\
\hline$\$ 20,000,000$ & $0.65 \%$ \\
\hline $30,000,000$ & $0.59 \%$ \\
\hline
\end{tabular}

S o u r c e: Average Financial Advisor Fees \& Costs 2017 Report, www.advisoryhq.com (accessed: 15.03.2017).

\section{THE OUTCOME OF THE RESEARCH PROCESS AND CONCLUSIONS}

Considering American retirement system challenges: low savings rates, shifting contribution responsibility from employer to employee, low IRA return rates, new solutions are more than desired. What is more, high management and fund fees are banes for investors. If changing retirement policies and regulations might not be an option, the cure for the system should be find in low cost retirement solutions. This paper proofs that replacing traditional mutual funds with ETFs can bring significant savings in fees, without impacting the performance. Choosing equity S\&P500 ETFs over mutual funds saves $0.45 \%$ in fees annually. What is more, if traditional FA will be replaced by robo advisor, additional savings can be made. Taking in consideration $\$ 100,000$ portfolio, shift to digital advice saves $0.87 \%$ in fees. Combining robo advisors savings with those brought by ETFs, potentially $1.32 \%$ annually can be saved, what brings $\$ 1,320$, that can remain on the account. Considering usually more than 
30 years investment horizon and instant growth of the portfolio, savings can be tremendous. ETFs are on the market for more than 20 years right now. Further steady growth of this segment probably will result in displacing mutual funds. The future of robo advisors looks bright as well. Digital advice will become a new standard for mass affluent customers. Not only American retirement system needs new solutions. Polish retirement system has own challenges as well. Poles social awareness of retirement issues and trust to government institutions is very low. One of the youngest in Europe retirement age connected with demographical challenges and lately disassembly of OFE brought a gap in Polish retirement system which hasn't been filled yet. Return rates of retirement solutions offered by mutual funds are mostly eaten up by fees. What is more, currently on GPW only 3 ETFs are listed and no digital advisory platform is available in Poland. Considering above arguments, there is definitely a niche for innovative low cost retirement solutions and further research on that topic.

\section{REFERENCES}

Aubry, J., Crawford, C., \& Munnell, A. (2015). Investment returns: Defined Benefit vs. Defined Contribution Plans, Center for Retirement Research Boston College.

Average Financial Advisor Fees \& Costs 2017 Report, http://www.advisoryhq.com (accessed: 15.03.2017).

Bernstein, J., \& Phyllis, A. (2002). Primer on Exchange-Traded Funds, Journal of Accountancy, Vol. 193, 38-41.

Brinson, G., \& Siegel, L. (2015). After 70 Years of Fruitful Research, Why Is There Still a Retirement Crisis? CFA Institute.

Dapp, F. (2016). FinTech - Traditionelle Banken als digitale Plattformen und Teil eines Finanz-Ökosystems, Springer.

Dellva, W. (2001). Exchange-Traded Funds Not for Everyone, Journal of Financial Planning, Vol. 14, 110-124.

Erickson, J., \& Madland, D. (2014). Fixing the drain on retirement savings, Center of American Progress.

France-Massey, T. (2016). The impact of robo advisors on institutional investment banking, Thinking Ahead, 27-31.

Gastineau, L. Gary (2001). Exchange-Traded Funds: An Introduction, Journal of Portfolio Management, Vol. 27.

Hill, J., Nadig, D., \& Hougan, M. (2015). A Comprehensive Guide to Exchange-Traded Funds (ETFs), CFA Institute.

Kostovetsky, L. (2003). Index Mutual Funds and Exchange Traded Funds, Journal of Portfolio Management, Vol. 29, 80-92. http://doi.org/10.3905/jpm.2003.319897. 
Nowak, K. (2016). Implementation of alternative index weighting to Warsaw Stock Exchange, Copernican Journal of Finance and Accounting, Vol 5, No 2, 166-179. http:// dx.doi.org/10.12775/CJFA.2016.021.

Poterba, M., \& Shoven J. (2002). Exchange Traded Funds: A New Investment Option for Taxable Investors, American Economic Review, Vol. 92, 422-427. http://doi. org/10.3386/w8781.

Robo-advisor - automation to enhance financial advisory, http://www.infosys.com (accessed: 12.03.2017).

Sironi, P. (2016). FinTech Innovation: From Robo-advisors to Goal-based Investing and Gamification, 2016.

Xia, Y. (2016). Debunking the myths behind the robo advisors, Thinking Ahead, 55-57.

http://www.etf.com (accessed: 15.03.2017).

http://www.etfdb.com (accessed: 15.03.2017).

http://www.statista.com (accessed: 15.03.2017).

http://us.pensiontracker.org (accessed: 15.03.2017). 